\title{
A New Variant of B-Spline for the Solution of Modified Fractional Anomalous Subdiffusion Equation
}

\author{
M. S. Hashmi $\mathbb{D}^{1}{ }^{1}$ Zainab Shehzad, ${ }^{1}$ Asifa Ashraf, ${ }^{2}$ Zhiyue Zhang, ${ }^{2}$ Yu-Pei Lv $\mathbb{D}^{3}$ \\ Abdul Ghaffar $\mathbb{D}^{4},{ }^{4}$ Mustafa Inc $\mathbb{D}^{1},{ }^{5,6,7}$ and Ayman A. Aly \\ ${ }^{1}$ Department of Mathematics, The Govt. Sadiq College Women University, Bahawalpur, Pakistan \\ ${ }^{2}$ School of Mathematical Sciences, Jiangsu Key Laboratory for NSLSCS, Nanjing Normal University, Nanjing 210023, China \\ ${ }^{3}$ Department of Mathematics, Huzhou University, Huzhou 313000, China \\ ${ }^{4}$ Department of Mathematics, Ghazi University, D G Khan, Pakistan \\ ${ }^{5}$ Department of Computer Engineering, Biruni University, Istanbul, Turkey \\ ${ }^{6}$ Department of Mathematics, Science Faculty, Firat University, 23119 Elazig, Turkey \\ ${ }^{7}$ Department of Medical Research, China Medical University Hospital, China Medical University, Taichung, Taiwan \\ ${ }^{8}$ Department of Mechanical Engineering, College of Engineering, Taif University, P.O.Box 11099, Taif 21944, Saudi Arabia
}

Correspondence should be addressed to Yu-Pei Lv; peipei@zjhu.edu.cn and Mustafa Inc; minc@firat.edu.tr

Received 11 May 2021; Accepted 18 June 2021; Published 5 July 2021

Academic Editor: Nehad Ali Shah

Copyright (C) 2021 M. S. Hashmi et al. This is an open access article distributed under the Creative Commons Attribution License, which permits unrestricted use, distribution, and reproduction in any medium, provided the original work is properly cited.

\begin{abstract}
The objective of this paper is to present an efficient numerical technique for solving time fractional modified anomalous subdiffusion equation. Anomalous diffusion equation has its role in various branches of biological sciences. B-spline is a piecewise function to draw curves and surfaces, which maintain its degree of smoothness at the connecting points. B-spline provides an active process of approximation to the limit curve. In current attempt, B-spline curve is used to approximate the solution curve of time fractional modified anomalous subdiffusion equation. The process is kept simple involving collocation procedure to the data points. The time fractional derivative is approximated with the discretized form of the Riemann-Liouville derivative. The process results in the form of system of algebraic equations, which is solved using a variant of Thomas algorithm. In order to ensure the convergence of the procedure, a valid method named Von Neumann stability analysis is attempted. The graphical and tabular display of results for the illustrated examples is presented, which stamped the efficiency of the proposed algorithm.
\end{abstract}

\section{Introduction}

The current study deals with the investigation of modified fractional anomalous subdiffusion equation using hybrid Bspline-based collocation method. The general form of the equation is

$\frac{\partial \psi(\xi, \tau)}{\partial \tau}=\sum_{i=1}^{n}\left(D_{\tau}^{1-\alpha_{i}}\right) \frac{\partial^{2} \psi(\xi, \tau)}{\partial \xi^{2}}+g(\xi, \tau) a \leq \xi \leq b, 0<\tau \leq \tau_{f}$,

with BC

$$
\begin{aligned}
\psi\left(\xi_{0}, \tau\right) & =p_{0}(\tau), \\
\psi\left(\xi_{N}, \tau\right) & =p_{1}(\tau),
\end{aligned}
$$

and IC

$$
\psi(\xi, 0)=q_{0}(\xi)
$$

where $D_{\tau}^{1-\alpha_{i}}$ is the Riemann-Liouville version of the fractional partial derivatives of order $\left(1-\alpha_{i}\right), \alpha_{i}{ }^{\prime} s$ are the fractional parameters with $1 \geq \alpha_{i}>0$ for $i=1,2, \cdots, n$ are fractional parameters appears in the equation. Here, $g_{0}(\tau), g_{1}(\tau), h_{0}$ $(\xi)$, and $h_{1}(\xi)$ are smooth functions, and $\xi, \tau$ are space and time variables.

Fractional PDEs have implementation in numerous fields of engineering and science. Some of them are placed in the great book of Podlubny [1] and in some recent work can be found in [2-6]; their applications can be found in the field of electromagnetic and mechanical engineering. 
Liu et al. [7] proposed a finite difference technique for the modified anomalous subdiffusion equation. Liu et al. [8] presented two versions of finite element approximation with semi- and full discretizations in a finite domain and compact difference scheme by Wang et al. [9]. An unconditionally stable high-order scheme was proposed by Mohebbi et al. [10]. The proposed scheme has a nonlinear source term. In another paper by Mohebbi et al. [11], the solution of 2D modified anomalous subdiffusion equation was proposed, and this method was based on radial basis functions.

Dag et al. [12] presented a numerical solution of Burger's equation using B-spline collocation method. Zahra and Elkholy [13] use cubic splines to represent a numerical solution of fractional differential equations. Nonclassical diffusion problems by Ismail et al. [14], advection-diffusion problems by Nazir et al. [15], and fractional subdiffusion equation by Zhu et al. [16] are solved by using B-spline collocation methods. In recent times, Hashmi et al. $[17,18]$ has solved Hunter Saxton equation and space fractional PDE by cubic trigonometric and hybrid B-spline method. The numerical solution of time-space fractional PDEs using B-spline wavelet method is presented by Kargar and Saeedi [19].

In present research, numerical formulation of the Riemann-Liouville derivative for anomalous subdiffusion equation described by Dehghan et al. [20] is used as time fractional derivative which is given as

$$
D_{\tau}^{1-\alpha} \psi(\xi, \tau)=\frac{\partial}{\partial \tau} I_{0+}^{\alpha} \psi(\xi, \tau)
$$

where

$$
\begin{aligned}
& \frac{I_{0+}^{\alpha} \psi\left(\xi, \tau^{n+1}\right)-I_{0+}^{\alpha} \psi\left(\xi, \tau^{n}\right)}{d \tau} \\
& \quad=\frac{d \tau^{\alpha}}{\Gamma(1+\alpha)}\left[\psi\left(\xi, \tau^{n}+1\right)+\sum_{p=0}^{n-1}\left(w_{p+1}-w_{p}\right) \psi\left(\xi, \tau^{n-p}\right)\right]+R^{\alpha},
\end{aligned}
$$

in which

$$
\left|R^{\alpha}\right| \leq C w_{p} d \tau+\alpha, 1=w_{0}>w_{1}>\cdots>w_{p}>0
$$

and $w_{p}=(p+1)^{\alpha}-p^{\alpha}$. Modified fractional anomalous subdiffusion equation have not been solved by hybrid B-spline collocation method yet. So, we solved modified fractional equation using hybrid B-spline collocation method. The proposed method is evaluated by Von Neumann stability analysis for convergence, which stamped that iterative method is stable without any condition over the domain. Numerical examples have been tested to highlight the performance of the numerical method. Numerical results are presented through tables and graphs. It shows that result very much agree with closed form of solution.

The paper is organized as follows: the description of proposed method along with methodology is presented in Section 2. In Section 3, initial state is calculated. Section 4 presents the convergence analysis of the iterative method.
TABLE 1: Values of hybrid function $H_{3, j}(\xi)$.

\begin{tabular}{lccccc}
\hline$\xi$ & $\xi_{j-2}$ & $\xi_{j-1}$ & $\xi_{j}$ & $\xi_{j+1}$ & $\xi_{j+2}$ \\
\hline$H_{3, j}$ & 0 & $Q_{1}$ & $Q_{2}$ & $Q_{1}$ & 0 \\
$H_{3, j}^{\prime}$ & 0 & $Q_{3}$ & 0 & $-Q_{3}$ & 0 \\
$H_{3, j}^{\prime}$ & 0 & $Q_{4}$ & $Q_{5}$ & $Q_{4}$ & 0 \\
\hline
\end{tabular}

TABLE 2: The numerical display of error of Example 1 when $N=50$, $\alpha_{1}=0.3, \alpha_{2}=0.7$, and $\tau_{f}=0.1$ for different values of $\gamma$.

\begin{tabular}{lccc}
\hline$\xi$ & $\gamma=0$ & $\gamma=0.5$ & $\gamma=1$ \\
\hline 0.10 & $6.71631 \times 10^{-4}$ & $6.71643 \times 10^{-4}$ & $6.71645 \times 10^{-4}$ \\
0.30 & $1.66881 \times 10^{-3}$ & $1.66884 \times 10^{-4}$ & $1.66885 \times 10^{-4}$ \\
0.50 & $2.12015 \times 10^{-3}$ & $2.12019 \times 10^{-4}$ & $2.12020 \times 10^{-3}$ \\
0.70 & $1.90480 \times 10^{-3}$ & $1.90484 \times 10^{-3}$ & $1.90484 \times 10^{-3}$ \\
0.90 & $8.75119 \times 10^{-3}$ & $8.75133 \times 10^{-3}$ & $8.75136 \times 10^{-3}$ \\
\hline
\end{tabular}

The accuracy of the iterative scheme is shown in Section 5, and Section 6 contains the conclusion.

\section{Description of Proposed Method}

Hybrid B-spline collocation method is utilized to resolve the modified anomalous subdiffusion equation. The approximate solution $\psi(\xi, \tau)$ to the analytical solution $\bar{u}(\xi, \tau)$ is considered as

$$
\psi(\xi, \tau)=\sum_{j=-1}^{N+1} \varsigma_{j}(\tau) H_{3, j}(\xi) .
$$

Here, time-dependent unknowns are denoted by $\varsigma_{j}(\tau)$, and $H_{3, j}(\xi)$ is a hybrid cubic B-spline basis function of third order and given as

$$
H_{3, j}(\xi)=\gamma B_{3, j}(\xi)+(1-\gamma) T_{3, j}(\xi)
$$

The value of $\gamma$ plays a very significant role in the hybrid cubic basis function. The hybrid nature of the proposed method occurred in $0<\gamma<1$, and at boundaries of this hybrid parameter, it produces trigonometric and polynomial B-spline. The nodal values are placed in Table 1.

$$
\begin{aligned}
& Q_{1}=\frac{\gamma}{6}+(1-\gamma) a_{1}, \\
& Q_{2}=\frac{2 \gamma}{3}+(1-\gamma) a_{2}, \\
& Q_{3}=\frac{\gamma}{2 h}+(1-\gamma) a_{3}, \\
& Q_{4}=\frac{\gamma}{h^{2}}+(1-\gamma) a_{5}, \\
& Q_{5}=-\frac{2 \gamma}{h^{2}}+(1-\gamma) a_{6} .
\end{aligned}
$$


TABLe 3: The numerical display of the solutions for Example 1 at different values of $\tau_{f}$ with $N=50$ and $\alpha_{1}=0.2, \alpha_{2}=0.8$.

\begin{tabular}{lcccccc}
\hline$\xi$ & Exact & $\begin{array}{c}\tau_{f}=0.1 \\
\text { Numerical }\end{array}$ & Error & Exact & Numerical & $\begin{array}{c}\tau_{f}=0.01 \\
\text { Error }\end{array}$ \\
\hline 0.10 & 0.0110517 & 0.0117205 & $6.68809 \times 10^{-4}$ & 0.0001105 & 0.0001172 & $6.6956 \times 10^{-6}$ \\
0.30 & 0.0134986 & 0.0151604 & $1.66181 \times 10^{-4}$ & 0.0001349 & 0.0001516 & $1.6637 \times 10^{-5}$ \\
0.50 & 0.0164872 & 0.0185985 & $2.11125 \times 10^{-3}$ & 0.0001649 & 0.0001860 & $2.1136 \times 10^{-5}$ \\
0.70 & 0.0201375 & 0.0220343 & $1.89679 \times 10^{-3}$ & 0.0002014 & 0.0002204 & $1.8989 \times 10^{-5}$ \\
0.90 & 0.0245960 & 0.0254675 & $8.71428 \times 10^{-4}$ & 0.0002460 & 0.0002547 & $8.7240 \times 10^{-6}$ \\
\hline
\end{tabular}

Here, $H_{3, j-1}\left(\xi_{j}\right), H_{3, j}\left(\xi_{j}\right)$, and $H_{3, j+1}\left(\xi_{j}\right)$ are only three nonzero basis functions that are included over subinterval $\left[\xi_{j}, \xi_{j+1}\right]$ owing to the local support property of B-spline basis function. Hence, the approximate solution and its derivatives with respect to $\xi$ at $\left(\xi_{j}, \tau^{n}\right)$, by using (8), are

$$
\begin{aligned}
\psi_{j}^{n} & =Q_{1} \varsigma_{j-1}^{n}+Q_{2} \varsigma_{j}^{n}+Q_{1} \varsigma_{j+1}^{n}, \\
\left(\psi_{x}\right)_{j}^{n} & =Q_{3} \varsigma_{j-1}^{n}-Q_{3} \varsigma_{j+1}^{n}, \\
\left(\psi_{x x}\right)_{j}^{n} & =Q_{4} \varsigma_{j-1}^{n}+Q_{5} \varsigma_{j}^{n}+Q_{4} \varsigma_{j+1}^{n} .
\end{aligned}
$$

2.1. Numerical Formulation. In order to apply the hybrid B-spline collocation method, we take (1) at $\left(\xi_{j}, t^{n}\right)$ and can be written in a form as

$$
\begin{aligned}
\left.\frac{\partial \psi(\xi, \tau)}{\partial \tau}\right|_{\xi=\xi_{j}}{ }_{\tau=\tau^{n}}= & \left(D_{\tau}^{1-\alpha_{1}} \psi\left(\xi_{j}, \tau^{n}\right)+D_{\tau}^{1-\alpha_{2}} \psi\left(\xi_{j}, \tau^{n}\right)\right) \\
& \cdot \frac{\partial^{2} \psi\left(\xi_{j}, \tau^{n}\right)}{\partial \xi^{2}}+g\left(\xi_{j}, \tau^{n}\right)
\end{aligned}
$$

Now, using the above discretized form of forward difference scheme and Riemann-Liouville derivative as a time fractional derivative will have the form

$$
\frac{\psi_{j}^{n+1}-\psi_{j}^{n}}{d \tau}=D_{\tau}^{1-\alpha_{1}}\left(\psi_{\xi \xi}\right)_{j}^{n}+D_{\tau}^{1-\alpha_{2}}\left(\psi_{\xi \xi}\right)_{j}^{n}+g\left(\xi_{j}, \tau^{n}\right)
$$

Simplifying the equation, we get

$$
\begin{gathered}
{\left[\left(Q_{1} \varsigma_{j-1}^{n+1}+Q_{2} \varsigma_{j}^{n+1}+Q_{1} \varsigma_{j+1}^{n+1}\right)+\left(Q_{1} \varsigma_{j-1}^{n}+Q_{2} \varsigma_{j}^{n}+Q_{1} \varsigma_{j+1}^{n}\right)\right]} \\
=d \tau(\kappa+\lambda)\left[\left(Q_{4} \varsigma_{j-1}^{n+1}+Q_{5} \varsigma_{j}^{n+1}+Q_{4} \varsigma_{j+1}^{n+1}\right)\right] \\
+d \tau(\kappa+\lambda)\left[\sum_{p=0}^{n-1}\left(d_{p}\right)^{\alpha_{1}}+\left(d_{p}\right)^{\alpha_{2}}\right] \\
\cdot\left(Q_{4} \varsigma_{j-1}^{n-p}+Q_{5} \varsigma_{j}^{n-p}+Q_{4} \varsigma_{j+1}^{n-p}\right)
\end{gathered}
$$

where $w_{p+1}-w_{p}=\left(d_{p}\right)^{\alpha_{i}}, \kappa=\left((d \tau)^{\alpha_{1}}\right) /\left(\Gamma\left(1+\alpha_{1}\right)\right)$ and $\lambda=$ $\left((d \tau)^{\alpha_{2}}\right) /\left(\Gamma\left(1+\alpha_{2}\right)\right)$

\section{Initial State}

The numerical procedure of the iterative process can be initiated using initial vector $\varsigma^{0}=\left[\varsigma_{-1}^{0}, \varsigma_{0}^{0}, \cdots \cdots, \varsigma_{N+1}^{0}\right]^{T}$. Initial conditions have been utilized to attain the initial vector by using the following procedure:

$$
\psi_{N}(\xi, 0)=\sum_{j=-1}^{N+1} \varsigma_{j}(0) B_{3, j}(\xi),
$$

where $\varsigma_{j}(0)^{\prime} s$ are unknown parameters. We need the initial approximation $\psi_{N}(\xi, 0)$ to satisfy the following conditions:

$$
\begin{aligned}
\psi\left(\xi_{j}, 0\right) & =q_{0}(\xi) j=0,1, \cdots, N, \\
\psi_{x}\left(\xi_{0}, 0\right) & =q_{0}^{\prime}(\xi) j=0, \\
\psi_{x}\left(\xi_{N}, 0\right) & =q_{0}^{\prime}(\xi) j=N .
\end{aligned}
$$

This produce a square matrix of order $(N+3)$ of the form

$$
Q \varsigma^{0}=B
$$

where $\varsigma^{0}=\left[\varsigma_{-1}^{0}, \varsigma_{0}^{0}, \cdots \cdots, \varsigma_{N+1}^{0}\right]^{T}$

$$
Q=\left(\begin{array}{cccccccc}
Q_{3} & 0 & -Q_{3} & \cdots & \cdots & \cdots & \cdots & 0 \\
Q_{1} & Q_{2} & Q_{1} & \ddots & & & & \vdots \\
0 & Q_{1} & Q_{2} & Q_{1} & \ddots & & & \\
\vdots & & & & \ddots & & & \vdots \\
\vdots & & & & & Q_{1} & Q_{2} & Q_{1} \\
0 & \cdots & \cdots & \cdots & \cdots & Q_{3} & 0 & -Q_{3}
\end{array}\right) \text {, }
$$

and $B=\left[q_{0}^{\prime}\left(\xi_{0}\right), q_{0}\left(\xi_{0}\right), \cdots, q_{0}\left(\xi_{N}\right), q_{0}^{\prime}\left(\xi_{N}\right)\right]^{T}$. The pentadiagonal system can be solved by the Thomas algorithm.

\section{Stability Analysis}

The stability of numerical technique can be examined by performing Von Neumann stability analysis. The stability of numerical schemes is closely related with numerical errors. 
TABle 4: The numerical display of errors for Example 1 at different values of $\alpha_{1}$ with $\alpha_{2}=0.3, N=70$, and $\tau_{f}=0.01$.

\begin{tabular}{lcccccc}
\hline$\xi$ & Exact & $\begin{array}{c}\alpha_{1}=0.1 \\
\text { Numerical }\end{array}$ & Error & Exact & \multicolumn{2}{c}{$\begin{array}{c}\alpha_{1}=0.5 \\
\text { Numerical }\end{array}$} \\
\hline 0.10 & 0.0017516 & 0.0018581 & $1.06557 \times 10^{-4}$ & 0.0002776 & 0.0002949 & $1.72452 \times 10^{-5}$ \\
0.30 & 0.0021394 & 0.0024041 & $2.64734 \times 10^{-4}$ & 0.0003391 & 0.0003819 & $4.28495 \times 10^{-5}$ \\
0.50 & 0.0026130 & 0.0029494 & $3.36343 \times 10^{-4}$ & 0.0004141 & 0.0004686 & $5.44384 \times 10^{-5}$ \\
0.70 & 0.0031916 & 0.0034938 & $3.02230 \times 10^{-4}$ & 0.0005058 & 0.0005547 & $4.89091 \times 10^{-5}$ \\
0.90 & 0.0038982 & 0.0040371 & $1.38893 \times 10^{-4}$ & 0.0006178 & 0.0006403 & $2.24703 \times 10^{-5}$ \\
\hline
\end{tabular}

TABLE 5: Numerical display of solutions of Example 1 of the TFPDE at different values of $N$ with $\alpha_{1}=0.6, \alpha_{2}=0.9$, and $\tau_{f}=0.001$.

\begin{tabular}{lcccccc}
\hline$\xi$ & Exact & $\begin{array}{c}N=70 \\
\text { Numerical }\end{array}$ & Error & Exact & Numerical & Error \\
\hline 0.10 & $3.495 \times 10^{-8}$ & $3.706 \times 10^{-8}$ & $2.11095 \times 10^{-9}$ & $3.495 \times 10^{-8}$ & $3.706 \times 10^{-8}$ & $2.10877 \times 10^{-9}$ \\
0.30 & $4.269 \times 10^{-8}$ & $4.793 \times 10^{-8}$ & $5.24514 \times 10^{-9}$ & $4.269 \times 10^{-8}$ & $4.793 \times 10^{-8}$ & $5.23974 \times 10^{-9}$ \\
0.50 & $5.214 \times 10^{-8}$ & $5.880 \times 10^{-8}$ & $6.66371 \times 10^{-9}$ & $5.214 \times 10^{-8}$ & $5.879 \times 10^{-8}$ & $6.65684 \times 10^{-9}$ \\
0.70 & $6.368 \times 10^{-8}$ & $6.967 \times 10^{-8}$ & $5.98679 \times 10^{-19}$ & $6.368 \times 10^{-8}$ & $6.966 \times 10^{-8}$ & $5.91222 \times 10^{-9}$ \\
0.90 & $7.778 \times 10^{-8}$ & $8.053 \times 10^{-8}$ & $2.75044 \times 10^{-9}$ & $7.778 \times 10^{-8}$ & $8.053 \times 10^{-8}$ & $2.74761 \times 10^{-9}$ \\
\hline
\end{tabular}

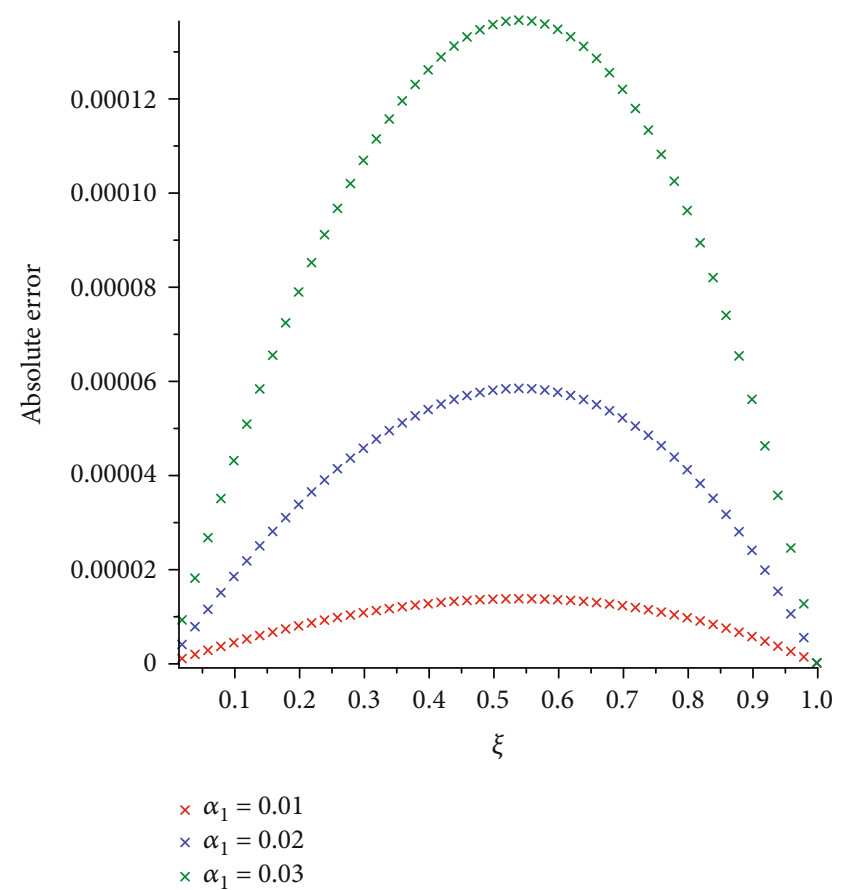

(a)

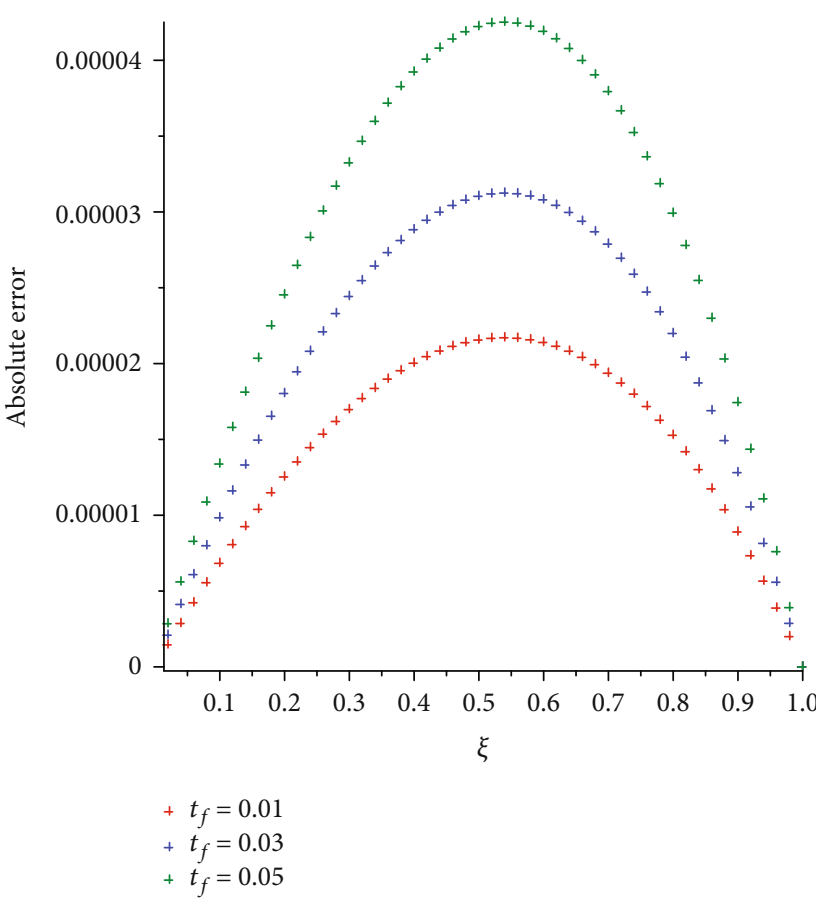

(b)

FIGURE 1: Numerical display of deviations from exact solution of Example 1 for various values of $\alpha_{1}$ (a) and for distinct values of $\tau_{f}$ (b).

The Von Neumann stability analysis provides us growth of the error in terms of Fourier series. For this, we used

$$
\varsigma_{l}^{n}=\eta_{n} e^{i l \theta h}
$$

in equation, where $\theta$ is a rational number and $i$ is a imaginary symbol. Utilizing (13), we get

$$
\begin{gathered}
{\left[\left(Q_{1} \varsigma_{l-1}^{n+1}+Q_{2} \varsigma_{l}^{n+1}+Q_{1} \varsigma_{l+1}^{n+1}\right)+\left(Q_{1} \varsigma_{l-1}^{n}+Q_{2} \varsigma_{l}^{n}+Q_{1} \varsigma_{l+1}^{n}\right)\right]} \\
=d \tau(\kappa+\lambda)\left[\left(Q_{4} \varsigma_{l-1}^{n+1}+Q_{5} \varsigma_{l}^{n+1}+Q_{4} \varsigma_{l+1}^{n+1}\right)\right]+d \tau(\kappa+\lambda) \\
\cdot\left[\sum_{p=0}^{n-1}\left(w_{p+1}-w_{p}\right)\left(Q_{4} \varsigma_{l-1}^{n-p}+Q_{5} \varsigma_{l}^{n-p}+Q_{4} \varsigma_{l+1}^{n-p}\right)\right]
\end{gathered}
$$




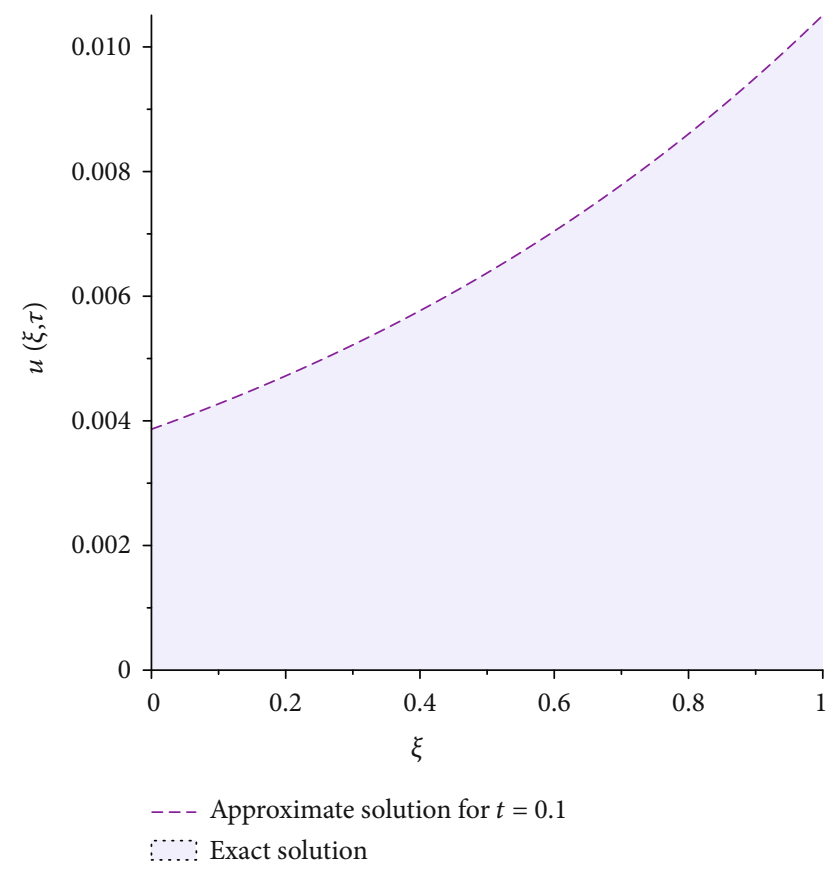

(a)

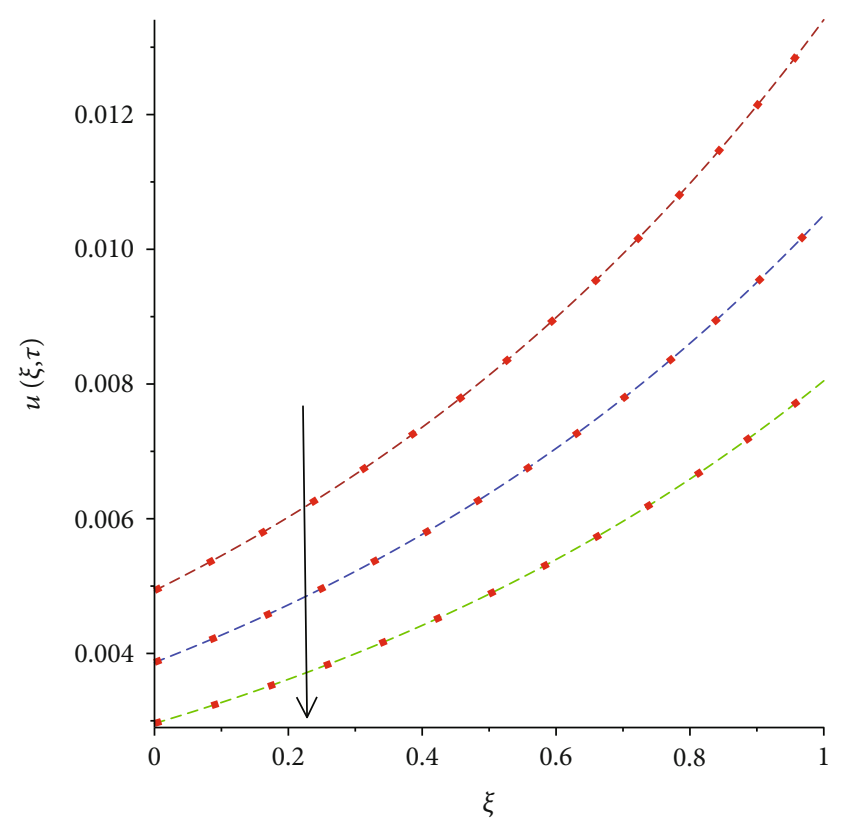

(b)

Figure 2: Graphical representation of exact and approximate solution for different values of $\xi$ in entire domain (a) and various values of $\tau_{f}$ i.e., $\tau_{f}=0.1,0.2,0.3$ in downward direction (b) of Example 1 .

Simplifying the equation in the presence of (18), we have

$$
\begin{aligned}
\eta_{n+1}[( & \left.\left.2 Q_{1} \cos (\theta h)+Q_{2}\right)-d \tau(\kappa+\lambda)\left(2 Q_{4} \cos (\theta h)+Q_{5}\right)\right] \\
= & \eta_{n}\left(2 Q_{1} \cos (\theta h)+Q_{2}\right)+d \tau(\kappa+\lambda) \\
& \cdot\left(2 Q_{4} \cos (\theta h)+Q_{5}\right)\left[\sum_{p=1}^{n-1}\left(d_{p}\right)^{\alpha_{1}}+\sum_{p=1}^{n-1}\left(d_{p}\right)^{\alpha_{2}}\right] \eta_{n-p} \\
& +\eta_{n}\left(2 Q_{4} \cos (\theta h)+Q_{5}\right) d \tau(\kappa+\lambda) .
\end{aligned}
$$

After simplification, we get

$$
\eta_{n+1}=v \eta_{n}+\mu\left[\sum_{p=1}^{n-1}\left(d_{p}\right)^{\alpha_{1}}+\sum_{p=1}^{n-1}\left(d_{p}\right)^{\alpha_{2}}\right] \eta_{n-p} .
$$

Thus, $\left|\eta_{n+1}\right| \leq\left|\eta_{n}\right|$.

Hence, our described method is stable unconditionally.

\section{Numerical Experiments}

In current section, numerical examples are computed to prove feasibility and accuracy of the scheme, and the results are displayed through graphs and tables.

Example 1. Consider the following problem

$$
\frac{\partial \psi(\xi, \tau)}{\partial \tau}=\left(D_{\tau}^{1-\alpha_{1}}+D_{\tau}^{1-\alpha_{2}}\right) \frac{\partial^{2} \psi(\xi, \tau)}{\partial \xi^{2}}+g(\xi, \tau)
$$

TABle 6: The absolute error of Example 2 when $N=70, \alpha_{1}=0.5$, $\alpha_{2}=0.2$, and $\tau_{f}=0.5$ for different values of $\gamma$.

\begin{tabular}{lccc}
\hline$\xi$ & $\gamma=0$ & $\gamma=0.3$ & $\gamma=1$ \\
\hline 0.10 & $1.79881 \times 10^{-4}$ & $1.78163 \times 10^{-4}$ & $1.77495 \times 10^{-4}$ \\
0.30 & $4.51450 \times 10^{-4}$ & $4.47218 \times 10^{-4}$ & $4.45572 \times 10^{-4}$ \\
0.50 & $5.72311 \times 10^{-4}$ & $5.66922 \times 10^{-4}$ & $5.64827 \times 10^{-4}$ \\
0.70 & $5.07258 \times 10^{-4}$ & $5.02359 \times 10^{-4}$ & $5.00454 \times 10^{-4}$ \\
0.90 & $2.27224 \times 10^{-4}$ & $2.24926 \times 10^{-4}$ & $2.24033 \times 10^{-4}$ \\
\hline
\end{tabular}

in the domain $0 \leq \xi \leq 1$ and for the interval $0<\tau \leq 1$, with the boundary conditions

$$
\psi(0, \tau)=\tau^{1+\alpha_{1}+\alpha_{2}}, \psi(1, \tau)=e\left(\tau^{1+\alpha_{1}+\alpha_{2}}\right),
$$

and the IC

$$
\psi(\xi, 0)=0,
$$

where

$$
\begin{aligned}
g(\xi, \tau)= & e^{\xi}\left[\left(1+\alpha_{1}\right) \tau^{\alpha_{1}}-\frac{\Gamma\left(2+\alpha_{1}+\alpha_{2}\right)}{\Gamma\left(1+2 \alpha_{1}+\alpha_{2}\right)} \tau^{2 \alpha_{1}+\alpha_{2}}\right. \\
& \left.-\frac{\Gamma\left(2+\alpha_{2}+\alpha_{2}\right)}{\Gamma\left(1+\alpha_{1}+2 \alpha_{2}\right)} \tau^{\alpha+2 \alpha_{2}}\right] .
\end{aligned}
$$


TABle 7: The numerical display of the solutions at different values of $\alpha$ with $N=40$ and $\tau_{f}=0.05$ for Example 2.

\begin{tabular}{lcccccc}
\hline$\xi$ & & $\alpha_{1}=0.3, \alpha_{2}=0.5$ & & & \multicolumn{2}{c}{$\alpha_{1}=0.3, \alpha_{2}=0.9$} \\
Numerical & Exact & Error \\
\hline 0.10 & 0.0348514 & 0.0353843 & $5.32893 \times 10^{-4}$ & 0.0262232 & 0.0256754 & $5.47808 \times 10^{-4}$ \\
0.30 & 0.0425676 & 0.0438695 & $1.29287 \times 10^{-3}$ & 0.0320291 & 0.0307043 & $1.32481 \times 10^{-3}$ \\
0.50 & 0.0519922 & 0.0536436 & $1.65143 \times 10^{-3}$ & 0.0391204 & 0.0374272 & $1.69323 \times 10^{-3}$ \\
0.70 & 0.0635034 & 0.0650357 & $1.53223 \times 10^{-3}$ & 0.0477818 & 0.0462044 & $1.57735 \times 10^{-3}$ \\
0.90 & 0.0775633 & 0.0783078 & $7.44550 \times 10^{-4}$ & 0.0583608 & 0.0575890 & $7.71809 \times 10^{-4}$ \\
\hline
\end{tabular}

TABle 8: The numerical display of solutions of Example 2 at different time level with $N=70, \alpha_{1}=0.5$, and $\alpha_{2}=0.7$.

\begin{tabular}{lcccccc}
\hline$\xi$ & Exact & $\begin{array}{c}\tau_{f}=0.01 \\
\text { Numerical }\end{array}$ & Error & Exact & $\begin{array}{c}\tau_{f}=0.001 \\
\text { Numerical }\end{array}$ & \begin{tabular}{c} 
Error \\
\hline 0.10
\end{tabular} \\
0.30 & 0.0015451 & 0.0015387 & $6.4223 \times 10^{-6}$ & 0.0000437 & 0.0000436 & $1.6572 \times 10^{-7}$ \\
0.50 & 0.0018872 & 0.0018728 & $1.4444 \times 10^{-5}$ & 0.0000534 & 0.0000531 & $3.1578 \times 10^{-7}$ \\
0.70 & 0.0023051 & 0.0022865 & $1.8558 \times 10^{-5}$ & 0.0000652 & 0.0000652 & $3.9949 \times 10^{-7}$ \\
0.90 & 0.0028154 & 0.0027968 & $1.8627 \times 10^{-5}$ & 0.0000797 & 0.0000792 & $4.4739 \times 10^{-7}$ \\
\hline
\end{tabular}

The exact solution is

$$
\bar{u}(\xi, \tau)=e^{\xi}\left(\tau^{1+\alpha_{1}+\alpha_{2}}\right)
$$

Table 2 describes the numerical errors at distinct rational values of $\gamma$, and it is evident that $\gamma=0$ is an appropriate solution than other case. So, trigonometric spline is the obvious choice to proceed further. In Table 3, the numerical display of the solutions at different values of $\tau_{f}$ is shown for $\alpha_{1}=$ $0.2, \alpha_{2}=0.8$, and $N=50$. Table 4 shows the numerical display of solution for different values of $\alpha_{1}$ at final time $\tau_{f}=$ 0.01 and $\alpha_{2}=0.3$. Table 5 shows the numerical display of solutions at different values of $N$ when $\alpha_{1}=0.6, \alpha_{2}=0.9$, and $\tau_{f}=0.001$. Figure 1(a) shows the graphical error when $\alpha_{1}$ varies at final time $\tau_{f}=0.05$ but $\alpha_{2}$ remains same. Figure 1(b) shows the graphical representation of errors at different terminal time $\tau_{f}$ when $\alpha_{1}$ and $\alpha_{2}$ remains same. Figure 2(a) represents the comparison of numerical and approximate solution in the entire domain; on the other hand, the comparison at different values of $\tau_{f}$ is presented in Figure 2(b).

Example 2. Consider the problem

$$
\frac{\partial \psi(\xi, \tau)}{\partial \tau}=\frac{1}{2}\left(D_{\tau}^{1-\alpha_{1}}+D_{\tau}^{1-\alpha_{2}}\right) \frac{\partial^{2} \psi(\xi, \tau)}{\partial \xi^{2}}+g(\xi, \tau),
$$

in the domain $0 \leq \xi \leq 1$ and for the time $0<\tau \leq 1$ with the $\mathrm{BC}$

$$
\begin{aligned}
& \psi(0, \tau)=\tau^{1+\alpha_{1}}+\tau^{1+\alpha_{2}}, \\
& \psi(1, \tau)=e\left(\tau^{1+\alpha_{1}}+\tau^{1+\alpha_{2}}\right),
\end{aligned}
$$

and the IC

$$
\psi(\xi, 0)=0
$$

where

$$
\begin{aligned}
g(\xi, \tau)= & e^{\xi}\left[\left(1+\alpha_{1}\right) \tau^{\alpha_{1}}-\frac{\Gamma\left(2+\alpha_{1}\right)}{\Gamma\left(1+2 \alpha_{1}\right)} \tau^{2 \alpha_{1}}\right. \\
& \left.+\left(1+\alpha_{2}\right) \tau^{\alpha_{2}}-\frac{\Gamma\left(2+\alpha_{2}\right)}{\Gamma\left(1+2 \alpha_{2}\right)} \tau^{2 \alpha_{2}}\right] .
\end{aligned}
$$

The exact solution is

$$
\bar{u}(\xi, \tau)=e^{\xi}\left(\tau^{1+\alpha_{1}}+\tau^{1+\alpha_{2}}\right) .
$$

In Table 6, the absolute error at different values of $\gamma$ is displayed, and it shows that $\gamma=0$ gives better result. Table 7 shows the numerical solution for different values of $\alpha_{2}$ with final time $\tau_{f}=0.05$ and $\alpha_{1}=0.3$. In Table 8 , the numerical display of solutions for different values of $\tau_{f}$ when $\alpha_{1}=0.5, \alpha_{2}=0.7$, and $N=70$ is presented. Figure 3(a) represents absolute error Example 2 of various values of $\alpha_{2}$ with fixed $\tau_{f}$. Figure $3(\mathrm{~b})$ describes the graphical representation of errors at various values of terminal time $\tau_{f}$ when $\alpha_{1}$ and $\alpha_{2}$ remains same. Figure 4(a) represents the comparison of numerical and approximate solution in entire domain; on the other hand, the comparison at different values of $\alpha_{1}$ is presented in Figure 4(b).

\section{Conclusion}

A B-spline collocation method is utilized to find the numerical approximation to the solution curve of fractional form of 


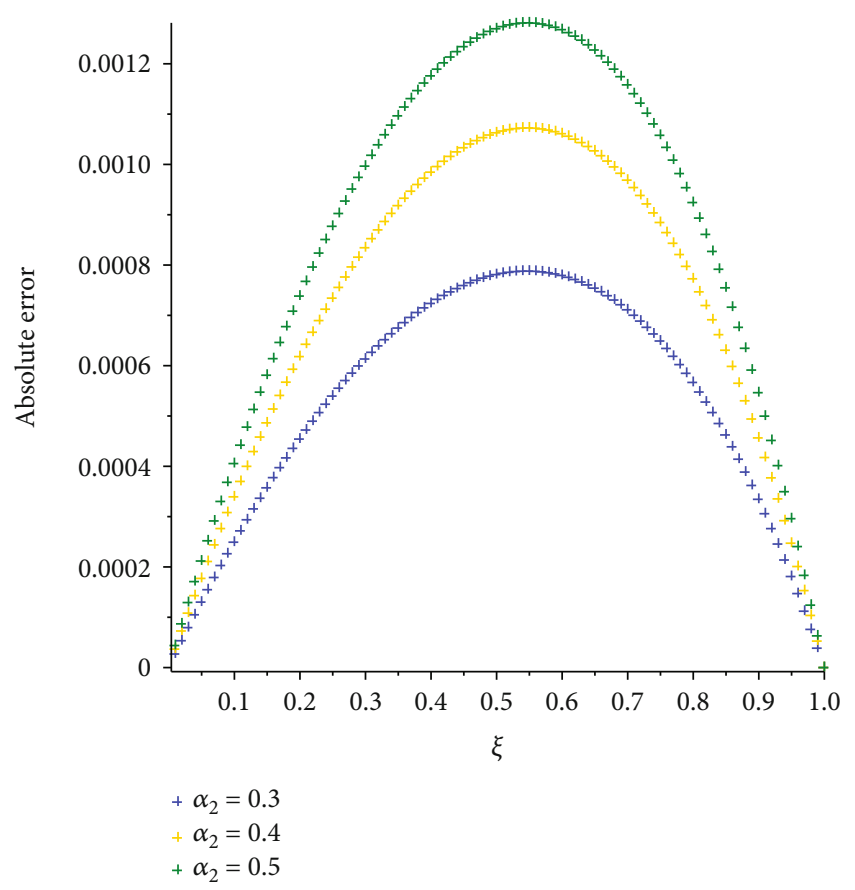

(a)

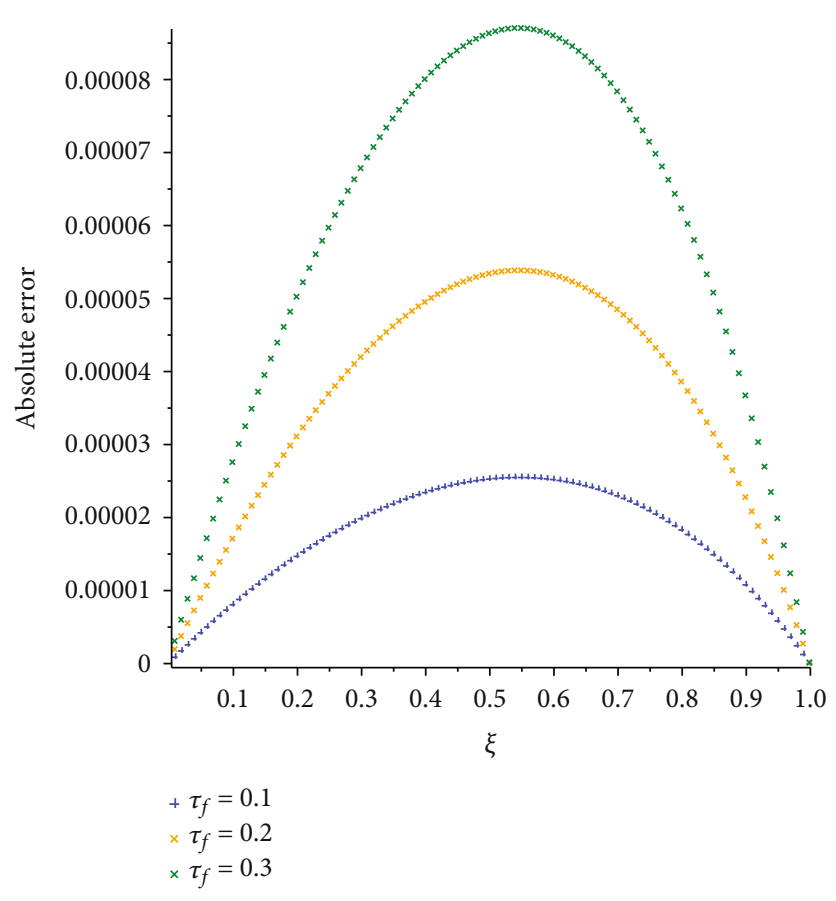

(b)

Figure 3: Graphical display of error for different values of $\alpha_{2}$ (a) and $\tau_{f}$ (b) Example 2.

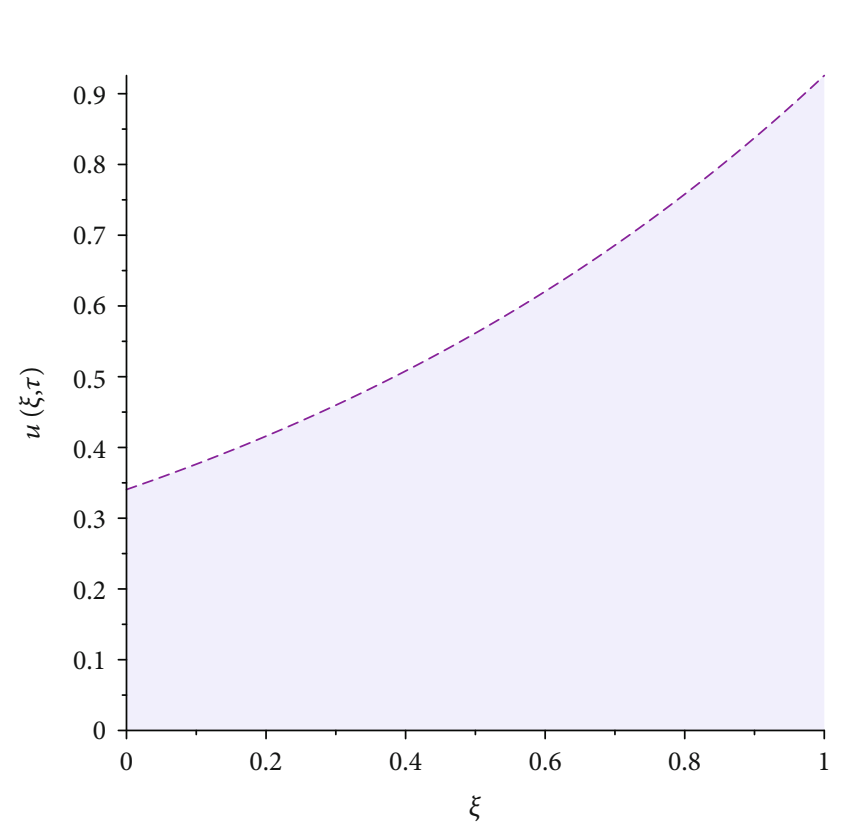

- - - Approximate solution for $t=0.1$

:....: Exact solution

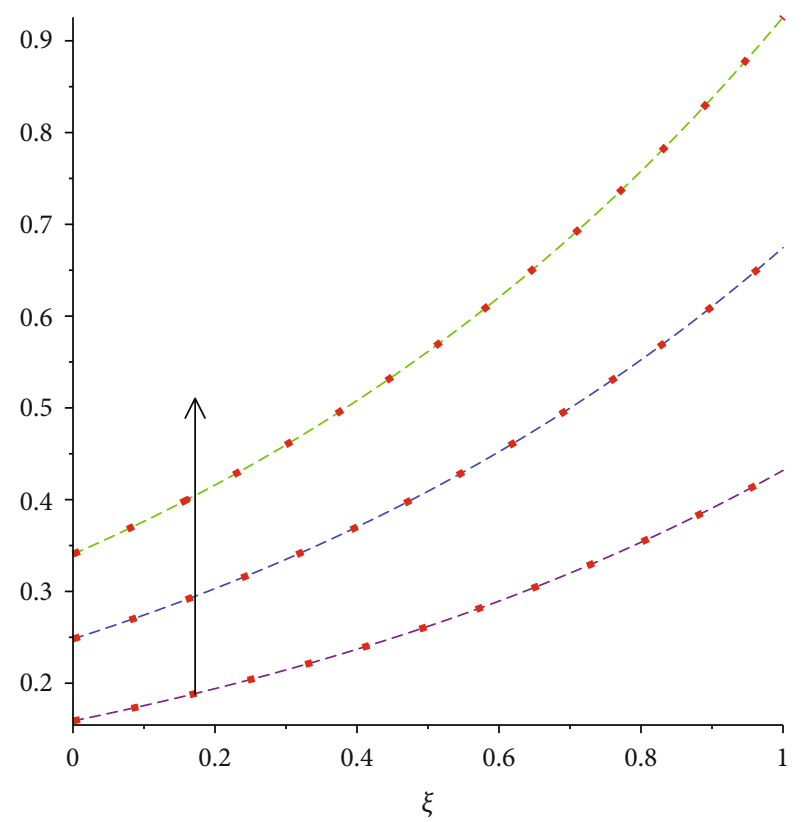

(b)

FIGURE 4: Graphical representation of exact and approximate solution for different values of $\xi$ in entire domain (a) and various values of $\alpha_{1}$ i.e., $\alpha_{1}=0.1,0.2,0.3$ in upward direction (b) of example 2 .

anomalous subdiffusion equation in modified form. First, formulate the formula for the modified fractional anomalous subdiffusion equation using discretized form of RiemannLiouville Derivative for the time fractional derivative. Then, we show the stability of B-spline method using the Fourier version of stability method, i.e., Von Neumann stability analysis. The iterative method is applied on numerical examples to show the applicability of described method. The graphs 
and tables demonstrate the numerical results. It shows that results are very much in agreement with exact solution. The technique can be further extended to higher dimension and nonlinear fractional PDEs in future.

\section{Data Availability}

No data were used to support this study.

\section{Conflicts of Interest}

The authors have no conflict of interests regarding the publication of this paper.

\section{Acknowledgments}

This work is supported by the Higher Education Commission of Pakistan under the NRPU project grant number 9306/Punjab/NRPU/R\&D/HEC/2017 and by the Taif University Researchers Supporting Project number (TURSP2020/77), Taif University, Taif, Saudi Arabia. The research was supported by the National Natural Science Foundation of China (Grant nos. 11971142, 11871202, 61673169, 11701176, 11626101 , and 11601485).

\section{References}

[1] I. Podlubny, Fractional Differential Equations, Academic Press, 1999.

[2] K. B. Oldham and J. Spanier, The Fractional Calculus: Theory and Application of Differentiation and Integration to Arbitrary Order, Academic Press, 1974.

[3] R. Metzler and J. Klafter, "The restaurant at the end of the random walk: recent developments in the description of anomalous transport by fractional dynamics," Journal of Physics, vol. 37, no. 31, pp. R161-R208, 2004.

[4] M. K. A. Kaabar, F. Martinez, J. F. Gomez-Aguilar, B. Ghanbari, M. Kaplan, and H. Gunerhan, "New approximate analytical solutions for the nonlinear fractional Schrödinger equation with second-order spatio-temporal dispersion via double Laplace transform method," Mathematical Methods in the Applied Sciences, pp. 1-19, 2021.

[5] M. M. Matar, M. I. Abbas, J. Alzabut, M. K. A. Kaabar, S. Etemad, and S. Rezapour, "Investigation of the $p$-Laplacian nonperiodic nonlinear boundary value problem via generalized Caputo fractional derivatives," Advances in Difference Equations, vol. 2021, Article ID 68, 2021.

[6] J. Alzabut, A. Selvam, R. Dhineshbabu, and M. K. Kaabar, "The existence, uniqueness, and stability analysis of the discrete fractional three-point boundary value problem for the elastic beam equation," Symmetry, vol. 13, no. 5, p. 789, 2021.

[7] F. Liu, C. Yang, and K. Burrage, "Numerical method and analytical technique of the modified anomalous subdiffusion equation with a nonlinear source term," Journal of Computational and Applied Mathematics., vol. 231, no. 1, pp. 160176, 2009.

[8] Q. Liu, F. Liu, I. Turner, and V. Anh, "Finite element approximation for a modified anomalous subdiffusion equation," Applied Mathematical Modelling, vol. 35, no. 8, pp. 41034116, 2011.
[9] Z. Wang and S. Vong, "Compact difference schemes for the modified anomalous fractional sub-diffusion equation and the fractional diffusion-wave equation," Journal of Computational Physics, vol. 277, pp. 1-15, 2014.

[10] A. Mohebbi, M. Abbaszadeh, and M. Dehghan, "A high-order and unconditionally stable scheme for the modified anomalous fractional sub-diffusion equation with a nonlinear source term," Journal of Computational Physics, vol. 240, pp. 36-48, 2013.

[11] A. Mohebbi, M. Abbaszadeh, and M. Dehghan, "Solution of two-dimensional modified anomalous fractional sub-diffusion equation via radial basis functions (RBF) meshless method," Engineering Analysis with Boundary Elements, vol. 38, pp. 7282, 2014.

[12] I. Dag, D. Irk, and A. Sahin, "B-spline collocation methods for numerical solutions of the Burgers' equation," Mathematical Problems in Engineering., vol. 2005, no. 5, pp. 521-538, 2005.

[13] W. K. Zahra and S. M. Elkholy, "The use of cubic splines in the numerical solution of fractional differential equations," International Journal of Mathematics and Mathematical Sciences, vol. 2012, Article ID 638026, 16 pages, 2012.

[14] M. Abbas, A. A. Majid, A. I. M. Ismail, and A. Rashid, "Numerical method using cubic trigonometric B-spline technique for nonclassical diffusion problems," Abstract and Applied Analysis, vol. 2014, Article ID 849682, 11 pages, 2014.

[15] T. Nazir, M. Abbas, A. I. M. Ismail, A. A. Majid, and A. Rashid, "The numerical solution of advection-diffusion problems using new cubic trigonometric B-splines approach," Applied Mathathematical Modelling, vol. 40, no. 7-8, pp. 4586-4611, 2016.

[16] X. Zhu, Y. Nie, Z. Yuan, J. Wang, and Z. Yang, “An exponential B-spline collocation method for the fractional sub-diffusion equation," Advances in Difference Equations, vol. 2017, no. 1, Article ID 1328, 2017.

[17] M. S. Hashmi, M. Awais, A. Waheed, and Q. Ali, "Numerical treatment of Hunter Saxton equation using cubic trigonometric B-spline collocation method," AIP Advances, vol. 7, no. 9, article 095124, 2017.

[18] R. Shikrani, M. S. Hashmi, N. Khan et al., "An efficient numerical approach for space fractional partial differential equations," Alexandria Engineering Journal, vol. 59, no. 5, pp. 2911-2919, 2020.

[19] Z. Kargar and H. Saeedi, "B-spline wavelet operational method for numerical solution of time-space fractional partial differential equations," International Journal of Wavelets, Multiresolution and Information Processing, vol. 15, no. 4, article 1750034, 2017.

[20] M. Dehghan, M. Abbaszadeh, and A. Mohebbi, "Legendre spectral element method for solving time fractional modified anomalous sub-diffusion equation," Applied Mathematical Modelling, vol. 40, no. 5-6, pp. 3635-3654, 2016. 